\title{
Increased inositol phosphoglycan P-type in the second trimester in pregnant women with type 2 and gestational diabetes mellitus
}

\author{
Marco Scioscia ${ }^{1,2, *}$, Khalid Gumaa ${ }^{3}$, Luigi E. \\ Selvaggi ${ }^{4}$, Charles H. Rodeck ${ }^{2}$ and Thomas W. \\ Rademacher $^{1}$ \\ ${ }^{1}$ Department of Immunology and Molecular Pathology, \\ Molecular Medicine Unit, Royal Free and University \\ College Medical School, London, UK \\ ${ }^{2}$ Department of Obstetrics and Gynecology, Royal Free \\ and University College Medical School, London, UK \\ ${ }^{3}$ College of Medicine and Medical Sciences, Arabian \\ Gulf University, Manama, Kingdom of Bahrain \\ ${ }^{4}$ Department of Obstetrics and Gynecology, University \\ of Medical Science of Bari, Bari, Italy
}

\begin{abstract}
A progressive insulin resistant state develops throughout human pregnancy. Inositol phosphoglycan P-type (PIPG), a second messenger of insulin, was reported to negatively correlate with the degree of insulin resistance in non-pregnant diabetic subjects. Urinary levels of P-IPG were assessed in insulin resistant states during pregnancy such as gestational diabetes mellitus (GDM, $n=44$ ) and type 2 diabetes mellitus (type $2 \mathrm{DM}, \mathrm{n}=25$ ) and in 69 normal pregnant women. Urinary levels of P-IPG were higher in GDM than controls with a positive trend of release throughout normal pregnancy $(P<0.01)$. $P$-IPG excretion was higher in diabetic (GDM and type $2 \mathrm{DM}$ ) than in healthy women in the second trimester $(P<0.05)$. A higher P-IPG urinary excretion occurs during the second trimester in pregnant women with clinically evident insulin resistance with a positive association with poor glycemic control.
\end{abstract}

Keywords: Diabetes type 2; gestational diabetes mellitus; glycemic control; inositol phosphoglycan; pregnancy.

\footnotetext{
${ }^{*}$ Corresponding author:

Dr. Marco Scioscia

Department of Obstetrics and Gynecology

Sacro Cuore Don Calabria General Hospital

Via Don A. Sempreboni

5, 37024 Negrar (VR)

Italy

Tel.: +393498724206

Fax: + 390457500480

E-mail: marcoscioscia@gmail.com
}

Inositol phosphoglycan P-type (P-IPG) was highly expressed in normal human pregnancy with a further increase during preeclampsia $[4,5]$, a complication of pregnancy characterized by insulin resistance. P-IPG belongs to a family of phospholipid-derived second messengers of insulin that can stimulate glucose and lipid metabolism. These molecules seem to be involved in the pathophysiology of insulin resistance [1].

A case-control pilot study was carried out to assess the role of P-IPG in clinically evident insulin resistant states during pregnancy such as gestational diabetes mellitus (GDM) and type 2 diabetes mellitus (type 2 DM). This study was approved by the local Ethical Committee. Forty-four women with GDM, 26 pregnant women with type 2 DM (diagnoses made according to WHO criteria [2]), and 69 normal pregnant women with similar maternal age and gestational age were consecutively recruited (Table 1). A mid-stream urine specimen was collected once only from all women recruited for this study to reduce any known bias. In fact, a higher excretion of $\mathrm{P}$ IPG was demonstrated well before the clinical onset of preeclampsia [5]. P-IPG content of urinary specimens was blindly assessed in triplicate using a polyclonal antibody-based ELISA as previously described [5] and corrected for urinary creatinine. Power analysis indicated a power of 0.96 with an alpha of 0.05 and a population standard deviation of 0.1 .

Maternal BMI and birth weights were higher on average in the type $2 \mathrm{DM}$ group than controls $(\mathrm{P}<0.01)$. Fasting plasma glucose was significantly higher in the GDM and type 2 DM groups than in controls $(5.9 \pm 1.1$ and $6.6 \pm 1.5$ vs. $5.1 \pm 0.9 \mathrm{mmol} / \mathrm{L}$, respectively, $\mathrm{P}<0.01)$. The urinary excretion of $\mathrm{P}$-IPG was higher in women with GDM and type 2 DM compared with controls (Table 1; $P<0.01)$. A positive trend in $P-I P G$ excretion throughout pregnancy was found in the control group only $(r=0.51$, $P<0.01$ ). No association was found with $2 h$ postprandial glycemia. A higher release of P-IPG was found in diabetic subjects before 30 weeks' gestation compared to normal pregnancy (Figure 1), whereas the difference was lost in the third trimester, when insulin resistance is physiologically increased.

The development of a physiological insulin resistant state during pregnancy is considered to be a mechanism that allows a safe nutrient balance between the mother and the fetus. When this metabolic balance is disrupted, when a decreased maternal insulin sensitivity or increased insulin resistance exists, abnormalities of glucose 
Table 1 Patient characteristics, glycemic control, and pregnancy outcome (mean \pm SD) in gestational diabetes mellitus (GDM), type 2 diabetes mellitus (type $2 \mathrm{DM}$ ), and control groups. In the second part of the table is reported P-IPG urinary excretion in the three study groups and its correlation with clinical parameters.

\begin{tabular}{|c|c|c|c|}
\hline & GDM $(n=44)$ & Type 2 DM (n=25) & Controls $(n=69)$ \\
\hline Maternal age (years) & $33.3 \pm 4.7$ & $32.9 \pm 4.5$ & $33.6 \pm 5.4$ \\
\hline Pre-pregnancy BMI (kg/m²) & $23.2 \pm 3.0$ & $24.6 \pm 3.2^{*}$ & $22.2 \pm 2.8$ \\
\hline Nulliparous (n) & $18(40.9 \%)$ & $13(52.0 \%)$ & $26(37.7 \%)$ \\
\hline Ethnicity (Caucasian/other; n) & $29 / 15$ & $11 / 14$ & $40 / 29$ \\
\hline Smoking (n) & 31 & 11 & 35 \\
\hline Gestational age (weeks) & $30.6 \pm 6.5$ & $31.0 \pm 5.9$ & $30.7 \pm 6.0$ \\
\hline \multicolumn{4}{|l|}{ Glycemic control } \\
\hline Fasting plasma glucose (mmol/L) & $6.3 \pm 0.7^{*}$ & $6.6 \pm 1.5^{*}$ & $5.1 \pm 0.9$ \\
\hline Post-prandial glycemia (mmol/L) & $7.1 \pm 0.4$ & $7.3 \pm 0.9$ & - \\
\hline $\mathrm{HbA}_{1 \mathrm{c}}(\%)$ & $6.2 \pm 0.6$ & $6.6 \pm 0.8$ & - \\
\hline Gestational age at delivery (weeks) & $38.5 \pm 1.4$ & $38.3 \pm 1.7$ & $38.9 \pm 1.5$ \\
\hline Birth-weight (g) & $3290 \pm 392$ & $3503 \pm 376^{\star}$ & $3211 \pm 333$ \\
\hline \multicolumn{4}{|l|}{ P-IPG urinary excretion } \\
\hline Mean (\% of positive control) & 33.1 & $49.8^{\star, \star \star}$ & 27.5 \\
\hline Median (\% of positive control) & 27.7 & 51.8 & 25.1 \\
\hline SEM (\% of positive control) & 1.9 & 3.2 & 1.2 \\
\hline Range (\% of positive control) & $19.7-64.2$ & $5.6-76.0$ & $5.6-59.9$ \\
\hline \multicolumn{4}{|l|}{ Correlations } \\
\hline \multirow[t]{2}{*}{ Gestational age } & $r=-0.21$ & $r=-0.54$ & $r=0.51$ \\
\hline & $P=0.17$ & $P<0.01$ & $\mathrm{P}<0.01$ \\
\hline \multirow[t]{2}{*}{ Pre-pregnancy BMI } & $r=-0.04$ & $r=0.10$ & $r=-0.15$ \\
\hline & $P=0.77$ & $P=0.59$ & $P=0.40$ \\
\hline \multirow[t]{2}{*}{ Fasting plasma glucose } & $r=-0.02$ & $r=-0.28$ & $r=-0.18$ \\
\hline & $P=0.89$ & $P=0.18$ & $P=0.77$ \\
\hline \multirow[t]{2}{*}{ Post-prandial glycemia } & $r=0.25$ & $r=0.21$ & - \\
\hline & $P=0.09$ & $P=0.38$ & \\
\hline \multirow[t]{2}{*}{$\mathrm{HbA}_{1 \mathrm{c}}$} & $r=0.23$ & $r=0.22$ & - \\
\hline & $P=0.13$ & $P=0.36$ & \\
\hline \multirow[t]{2}{*}{ Birth-weight } & $r=-0.14$ & $r=-0.36$ & $r=0.20$ \\
\hline & $P=0.36$ & $P=0.13$ & $P=0.43$ \\
\hline
\end{tabular}

${ }^{*} \mathrm{P}<0.01$, compared with controls.

${ }^{\star *} \mathrm{P}<0.01$, type 2 diabetes mellitus (type $2 \mathrm{DM}$ ) vs. gestational diabetes mellitus (GDM).

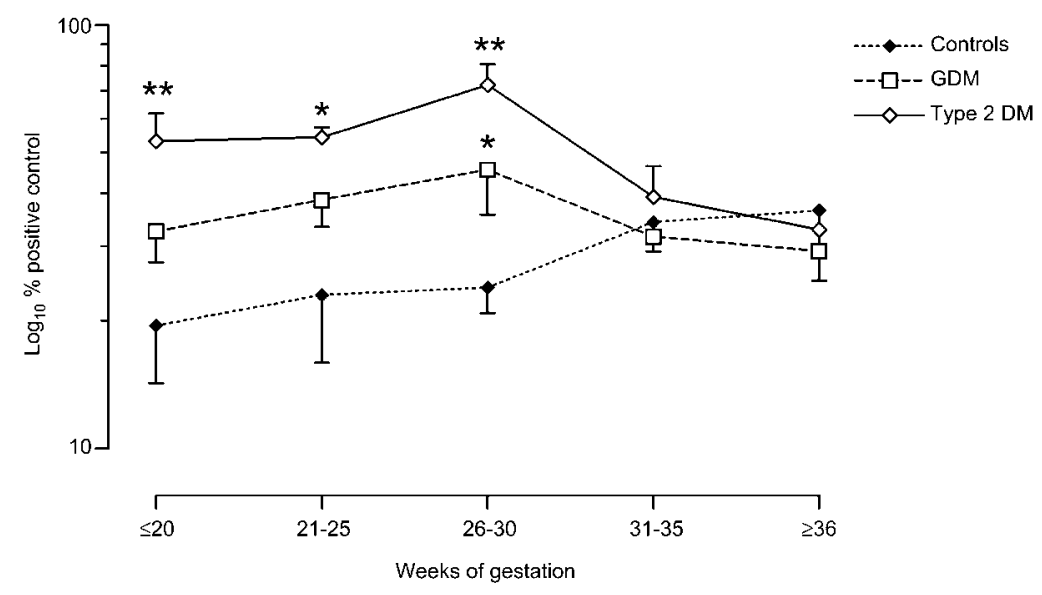

$\mathrm{n}\left\{\begin{array}{lrrrrr}\text { Controls } & 10 & 11 & 13 & 20 & 15 \\ \text { GDM } & 5 & 6 & 9 & 14 & 10 \\ \text { Type 2 DM } & 5 & 5 & 4 & 6 & 5\end{array}\right.$

Figure 1 P-IPG urinary excretion in the three study groups stratified by gestational age $(C=$ controls, GDM = gestational diabetes mellitus, type $2 \mathrm{DM}$ = type 2 diabetes mellitus). Values were divided into 5 -week spans and reported as mean and standard error. $\mathrm{P}$ IPG release was higher in GDM and type 2 DM groups between 21 and 30 weeks of gestation compared with controls ( ${ }^{\star} P<0.05$; $\left.{ }^{* *} \mathrm{P}<0.01\right)$. 
metabolism become clinically evident (GDM or type 2 $D M)$. The role of molecules with insulin-like characteristics stimulated the interest of many researchers. In diabetic subjects, a reduced P-IPG content in musclebiopsy and urinary specimens from insulin-resistant subjects was reported [1], suggesting that P-IPG has an important role in mediating the effects of insulin on peripheral glucose utilization.

Interestingly, we found a higher production/release of $P-I P G$ in the second trimester in women with glucose tolerance abnormalities (GDM and type $2 \mathrm{DM}$ ) compared to healthy women. The comparison to non-pregnant insulin-resistant subjects seems to support the hypothesis of the fetal/placental unit as a potential source of PIPG [4] which might partially restore insulin sensitivity and glucose disposal. In fact, Nestler and co-workers [3] reported that administration of chiro-inositol (P-IPG) increases insulin sensitivity in women with polycystic ovary syndrome, a disorder strongly associated with insulin resistance. The anabolic effect of P-IPG may explain the increased body fat and birth weight in fetuses from diabetic mothers compared to fetuses from normal pregnancies. Therefore, the fetal/placental unit seems not to have a simply permissive role in pregnancies with abnormal glucose metabolism; rather it adapts to the maternal environment and assumes an active role.

\section{References}

[1] Kennington AS, Hill CR, Craig J, Bogardus C, Raz I, Ortmeyer HK, et al. Low urinary chiro-inositol excretion in noninsulin-dependent diabetes mellitus. N Engl J Med. 1990; 323:373-8.

[2] Metzger BE, Coustan DR. Summary and recommendations of the Fourth International Workshop-Conference on Gestational Diabetes Mellitus. The Organizing Committee. Diabetes Care. 1998;21(Suppl 2):B161-7.

[3] Nestler JE, Jakubowicz DJ, Reamer P, Gunn RD, Allan G, Ovulatory and metabolic effects of D-chiro-inositol in the polycystic ovary syndrome. N Engl J Med. 1999;340: 1314-20.

[4] Scioscia M, Gumaa K, Whitten M, Selvaggi LE, Rodeck CH, Rademacher TW. Inositol phosphoglycan P-type in healthy and preeclamptic pregnancies. J Reprod Immunol. 2007;76: 85-90.

[5] Williams PJ, Gumaa K, Scioscia M, Redman CW, Rademacher TW. Inositol phosphoglycan P-type in preeclampsia: a novel marker? Hypertension. 2007;49:84-9.

The authors stated that there are no conflicts of interest regarding the publication of this article.

Received December 30, 2008. Accepted March 19, 2009. Previously published online June 3, 2009. 